\title{
Art-Neuroscience: poetic-artistic investigations on the impacts of Hostile Architecture on the homeostasis of users in the context of COVID-19 pandemic
}

\author{
Keywords \\ Hostile Architecture, Neurosciences, Contemporary Urban Art, Homeodynamic Environments and Products, \\ Pandemic and Post-pandemic Contexts.
}

This research is based on the theoretical and poetic-artistic production on the theme "Hostile Architecture", which comprises urban elements and practices that restrict the permanence of individuals in public space, such as metal spikes on walls or boulders under viaducts. The reason why these elements are placed in the public space is related to security and they serve to inhibit undesirable social behavior, although they promote fear and, as consequence, leave abandoned spaces in the city which become susceptible to violence. It mainly affects the homeless population, but also affects delivery men, people with low mobility, families with children, among others urban dynamics. By limiting urban shelter, these hostile urban elements and practices act as aggressive stimuli, which disturb the balance of the human organism and are intensified with the socio-economic aggravations intensely triggered by the COVID-19 pandemic. The initial focus of this study is the central region of São Paulo (the biggest city in Brazil and the most affected by the pandemic, with the highest number of cases and deaths) and a critical bias of such practices is proposed, especially regarding its impacts on the biological homeostasis of users in this region. Therefore, the research is based on cooperation between the fields of Architecture and Cognitive and Behavioral Neurosciences, especially in the concept of Homeodynamic Environments and Products. The research methodology comprises a transdisciplinary approach and involves four main stages of development: [a] literature review under the Hostile Architecture theme; [b] theoretical articulation between the proposed theme and the concepts of Cognitive and Behavioral Neurosciences, especially from the perspective of biological homeostasis concept; [c] field research aimed at the observation, recording, analysis and interpretation of users behavior in face of the stimuli caused by hostile urban elements/practices, as well as the application of unstructured interviews with the referred public, to find out how such elements affect their physical and mental states during the pandemic context. The material resulting from these analyzes is the basis for the concept and development of the artworks produced by the artist-author; and [d] conception and development of a physicaldigital poetic-artistic proposal. The poetic-artistic proposal is produced from two articulated approaches: the first is a digital platform for collective mapping of hostile architecture elements in Brazil territory as well as for discussion and scientific dissemination about the hostile architecture theme, through texts and posts on social networks. In this way, all collected material is organized in an interactive map with photos and their locations. The platform broadens critical perspectives and encourages reflections about the hostile architecture through drift and photographic action. The second one comprises the authorartist's poetic practice, elaborated as drawings, collages, three-dimensional objects and digital art. It is based on her immersion in each research development stage mentioned above, which aims to present and discuss new possibilities of presence and urban experience in pandemic and post-pandemic contexts. 